\title{
Effects of Rituximab Including Long-term Maintenance Therapy in Children with Nephrotic Syndrome in a Single Center of Korea
}

\author{
Seong Heon Kim, M.D. ${ }^{1,2}$ \\ Taek Jin Lim, M.D. ${ }^{1}$ \\ JiYeon Song, M.D. ${ }^{1}$ \\ SuYoung Kim, M.D., Ph.D. ${ }^{1,2}$ \\ Department of Pediatrics ${ }^{1}$, Pusan \\ National University Children's Hospital, \\ Yangsan, Korea, Research Institute for \\ Convergence of Biomedical Science \\ and Technology ${ }^{2}$, Pusan National \\ University Yangsan Hospital, Yangsan, \\ Korea
}

Corresponding author:

Su Young Kim, M.D. Ph.D.

Department of Pediatrics, Pusan National

University Children's Hospital, 20,

Geumo-ro, Mulgeum-eup, Yangsan-si, Gyeongsangnam-do, 50612, Republic of Korea

Tel: +82-55-360-2180

Fax: +82-55-360-2181

E-mail:suyung@pusan.ac.kr

Received: 6 March 2018

Revised: 23 March 2018

Accepted: 27 March 2018

\begin{abstract}
This is an open-access article distributed under the terms of the Creative Commons Attribution Non-Commercial License (http:// creativecommons.org/licenses/by-nc/4.0/) which permits unrestricted non-commercial use, distribution, and reproduction in any medium, provided the original work is properly cited.
\end{abstract}

Rituximab (RTX) is a chimeric monoclonal antibody that inhibits CD20-mediated B-cell proliferation and differentiation. Several studies have examined its use in intractable nephrotic syndrome (NS) with some positive results. However, those studies examined such effects for a short-term period of 1 year, and some patients continued to relapse after a lapse in RTX treatment. Our use of RTX as a maintenance therapy (RTX injection when the CD19 cell count exceeded 100-200/ $\mu \mathrm{L}$ before relapse) showed some noticeable efficacy. We used RTX in 19 patients with steroid-dependent NS (SDNS). In 12 patients treated with RTX maintenance therapy, only one relapse occurred. The mean treatment period was $23.4 \pm 12.7$ months, and the mean number of RTX administrations was 3.9 \pm 1.6 . The relapse rates were decreased (from 2.68/year to 0.04/year), and the drug-free period also increased (from 22.5 days/year to 357.1 days/year) during maintenance therapy. The other seven patients were treated with one cycle of RTX or additional cycles in case of relapse (non-maintenance therapy). Relapse rates were significantly decreased after RTX treatment (from 1.76/year to $0.96 /$ year, $P=0.017$ ). The relapsefree period was $15.55 \pm 7.38$ (range, 5.3-30.7) months. No severe side effects of RTX were found except for a hypersensitivity reaction such as fever and chills during its infusion. In conclusion, RTX is considered an effective and safe option to reduce the relapse rate by a single- or maintenance-interval therapy in SDNS.

Key words: Nephrotic syndrome; Children; Rituximab; Steroid-dependent

\section{Introductions}

Idiopathic nephrotic syndrome (NS) is one of the most common kidney diseases in children and most patients generally respond to steroid therapy (steroid-sensitive). However, $10-20 \%$ of these patients do not respond to steroids (steroid-resistant) and have a high risk of developing end-stage renal disease ${ }^{1)}$. Moreover, a significant portion of steroid-sensitive patients are likely to relapse frequently or become dependent on steroids and develop a high risk of steroid toxicity, including hypertension, growth disturbance, and glucose intolerance ${ }^{2}$. In cases of such intractable NS, several drugs such as cyclosporine, tacrolimus, levamisole, mycophenolate mofetil, and cyclophosphamide are used to reduce steroid toxicity or overcome steroid resistance. However, many of these drugs have significant side effects and are not always effective. 
Rituximab (RTX) is a chimeric monoclonal antibody that inhibits CD20-mediated B cell proliferation and differentiation. In the past 10 years, RTX has been used to treat patients with steroid-dependent or -resistant $\mathrm{NS}^{1,3-5)}$. The 2012 Kidney Disease: Improving Global Outcomes clinical practice guidelines introduced RTX as a treatment option for steroid-dependent NS.

Here we studied the efficacy of maintenance and nonmaintenance treatment with RTX in SDNS and examined the safety profile of RTX in a single center in Korea.

\section{Materials and methods}

\section{Study design and patients}

The subjects in this study were patients with SDNS who were treated with RTX and followed up for 6 months at Pusan National University Children's Hospital from November 2008 to October 2015. SDNS is defined as two or more relapses of NS during the reduction of steroid treatment or within 2 weeks of the discontinuation of steroid treatment.

RTX administration schedule was divided into two ways of maintenance and non-maintenance according to patients' willing. Maintenance therapy refers to more than two cycles of RTX treatment and RTX was administered before relapse when the CD 19 cell count increased more than $100-200 / \mu \mathrm{L}$. After 1st rituximab treatment, we provided maintenance therapy in patients who want regular use of rituximab before relapse. Until just before RTX maintenance therapy, all patients had received steroid treatment for more than one year and one to two years of CNI treatment.

As some patients with SDNS did not want regular RTX treatment, we used RTX only after relapse during steroid dependency in these patients (Non maintenance therapy). RTX was injected during the early tapering period with alternative day dosing of the steroid after achieving remission. An additional single RTX dose was administered to some patients with SDNS whose B cell count was not totally depleted despite RTX injection.

Rituximab therapy was approved by Institutional Review Board in Pusan National University Yansan Hospital.

\section{Method and statistics}

We retrospectively collected details including patient sex, age at diagnosis, age of starting treatment, number of treatment cycles, number of relapses and drugs free period in the maintenance therapy, number of relapses per year in the non-maintenance therapy. The efficacy of the nonmaintenance therapy was evaluated via the comparison of relapse rate before and during treatment using the Wilcoxon signed rank test performed using SPSS for Windows version 18.0 (SPSS Inc., Chicago, Illinois, USA). $P$ values were two-tailed and values of $P<0.05$ were considered statistically significant.

\section{Results}

A total of 19 patients with SDNS were enrolled and analyzed. The mean age at diagnosis was $56.4 \pm 36.7$ months, and the age of starting RTX treatment was $140.4 \pm 51.0$ months. The mean duration from diagnosis to RTX treatment was $85.3 \pm 55.9$ months (Table 1 ).

Among the 19 patients with SDNS, 12 were treated with maintenance therapy and seven were treated with nonmaintenance therapy. In non-maintenance therapy, relapse rates were significantly decreased after RTX treatment (from 1.76/year to $0.96 /$ year, $P=0.017$ ). The mean relapsefree period was $15.55 \pm 7.38$ (range, 5.3-30.7) months. Three patients in the non-maintenance therapy repeated two or more cycles because of frequent relapses. Two patients (patient No.13 and 19) achieved long-term remission for 24 months without additional RTX.

In maintenance therapy, only one relapse occurred in patient No.6. The mean treatment period was $23.4 \pm 12.7$ (range, 7-48) months and the mean number of treatments was 3.9 \pm 1.6 (range, $2-7$ ). The mean time interval between treatments was $6.23 \pm 1.65$ (range, 3.9-9.9) months. These patients did not take any other medication during maintenance period except one relapsed patients with 3-4 months of steroid therapy. Table 2 shows the efficacy of RTX maintenance therapy.

The most common side effect of RTX therapy was an infusion-related hypersensitivity reaction, such as fever and chills. However, the RTX infusion was completed in all patients by slowing down of the infusion rate and use of 
Table 1. Clinical Characteristics of 19 Patients with SDNS

\begin{tabular}{|c|c|c|c|c|c|c|}
\hline & Patient no. & Sex & Age at diagnosis (months) & Age at starting RTX (months) & RTX cycles* & RTXTx period (months) \\
\hline \multirow[t]{12}{*}{ Maintenance therapy } & 1 & $M$ & 39 & 222 & 5 & 35 \\
\hline & 2 & M & 70 & 99 & 3 & 14 \\
\hline & 3 & $\mathrm{~F}$ & 71 & 136 & 3 & 13 \\
\hline & 4 & M & 24 & 190 & 7 & 48 \\
\hline & 5 & M & 30 & 155 & 6 & 25 \\
\hline & 6 & M & 49 & 200 & 4 & 29 \\
\hline & 7 & $\mathrm{~F}$ & 19 & 100 & 5 & 26 \\
\hline & 8 & M & 102 & 141 & 2 & 11 \\
\hline & 9 & M & 65 & 152 & 5 & 40 \\
\hline & 10 & M & 22 & 52 & 2 & 14 \\
\hline & 11 & $F$ & 170 & 205 & 2 & 7 \\
\hline & 12 & M & 24 & 149 & 3 & 19 \\
\hline \multirow[t]{7}{*}{ Non-maintenance therapy } & 13 & M & 49 & 86 & 1 & \\
\hline & 14 & M & 59 & 119 & 3 & \\
\hline & 15 & M & 28 & 68 & 2 & \\
\hline & 16 & M & 36 & 218 & 1 & \\
\hline & 17 & M & 109 & 146 & 2 & \\
\hline & 18 & M & 65 & 148 & 1 & \\
\hline & 19 & M & 40 & 81 & 1 & \\
\hline
\end{tabular}

*One cycle refers to only a single infusion or a single infusion and an additional infusion in patients not achieving total B cell depletion despite a first RTX infusion.

Abbreviations: F, female; M, male; RTX, rituximab; SNS, steroid-dependent nephrotic syndrome.

Table 2. The Efficacy of RTX as Maintenance Therapy

\begin{tabular}{lccc}
\hline & Before RTX & During RTX & $P$ value \\
\hline $\begin{array}{l}\text { Relapse rate } \\
\text { (numbers/year) }\end{array}$ & 2.68 & 0.04 & $<0.01$ \\
$\begin{array}{l}\text { Drugs* free period } \\
\text { (days/year) }\end{array}$ & 22.5 & 357.1 & $<0.01$ \\
\hline
\end{tabular}

*Drugs include all immunosuppressive agents such as corticosteroid, calcineurin inhibitors and mycophenolate mofetil.

Abbreviation: RTX, rituximab.

Table 3. Side Effect Profiles of RTX

\begin{tabular}{lc}
\hline Side effect & No. \\
\hline Fever & $15(65.2 \%)$ \\
Chills & $14(60.9 \%)$ \\
Headache & $4(17.4 \%)$ \\
Chest discomfort & $4(17.4 \%)$ \\
Itching & $3(13.0 \%)$ \\
Nausea/vomiting & $2(8.7 \%)$ \\
Abdominal pain & $2(8.7 \%)$ \\
Myalgia & $1(4.2 \%)$ \\
Herpes zoster infection & $1(4.3 \%)$ \\
\hline
\end{tabular}

Abbreviation: RTX, rituximab.

medications including antihistamines and corticosteroids. Other side effects included headache, chest discomfort, and itching (Table 3).

\section{Discussion}

Here we studied the efficacy of RTX treatment for intractable NS including 19 patients with SDNS. Twelve patients were treated with maintenance RTX therapy and seven were treated with non-maintenance therapy. In both therapy, RTX was effective at maintaining remission without severe side effects.

RTX was first introduced for the treatment of B cell lymphoma. Thereafter, patients with several autoimmune diseases, such as rheumatoid arthritis or systemic lupus erythematosus, were treated with it, especially when their conditions were refractory to standard treatment ${ }^{6,7)}$. After Benz et al reported successful RTX treatment in SDNS with idiopathic thrombocytopenic purpura ${ }^{8)}$, there have been many reports of RTX treatment for refractory $\mathrm{NS}^{2,4,5,9,10)}$. Iijima et al recently performed a multicenter, double-blind, randomized, placebo-controlled trial of RTX therapy in patients with steroid-dependent NS, which demonstrated 
efficacy and safety ${ }^{11}$. Moreover, several studies on the effectiveness of RTX therapy for patients resistant to both steroids and CNI have been reported ${ }^{4,12,13)}$. However, a randomized controlled trial performed by Magnasco et al did not show a benefit of the addition of RTX to standard therapy consisting of prednisone and $\mathrm{CNI}^{14)}$.

In 2015, Bagga et al studied efficacy of RTX in patients with steroid-dependent, steroid-resistant, CNI-dependent, and CNI-resistant NS. They concluded that RTX is effective and safe in steroid-dependent and -resistant CNI-dependent NS but unsatisfactory in steroid- and CNI-resistant $\mathrm{NS}^{9}$. Focal segmental glomerulosclerosis was associated with a higher risk of non-response, while a lack of response was associated with progressive chronic kidney disease ${ }^{9)}$. Cravedi et al suggested that a low serum RTX level in patients with SRNS can be caused by urinary loss during the nephrotic state of disease ${ }^{15)}$.

Most nephrologists currently do not know exactly how frequently RTX administration should be repeated in NS. One to four doses were mostly used to maintain remission in SDNS in many studies ${ }^{11,16-18)}$. Iijima et al also used RTX at $375 \mathrm{mg} / \mathrm{m}^{2}$ once weekly for 4 weeks ${ }^{11)}$. However, $42 \%$ of the patients who received RTX four times had one or more serious side effects, although most were treatable and no patients died. Thus, Niu et al investigated the efficacy of a single dose of RTX that appeared to have fewer side effects in $\mathrm{SDNS}^{199}$. Among 19 patients, 10 remained in complete remission and did not relapse without oral steroids or immunosuppressants for 4-50 months despite recovery of the $B$ cell count. They concluded that RTX is an effective treatment for SDNS without significant side effects ${ }^{19}$. Fuginaga et al also demonstrated that a single dose of RTX allowed for a decreased dose of steroids in SDNS ${ }^{17}$. Another report demonstrated that RTX treatment intensity (one or two vs three or four doses) does not seem to influence the longterm remission rate. Eight of 37 patients remained in longterm remission after only one RTX infusion ${ }^{10}$.

Many patients with SDNS benefitted from RTX, but some relapsed after a lapse of time after RTX injection and returned to the previous state of steroid dependency. Thus, for those patients, we thought maintenance therapy with RTX would prevent relapses. Tellier et al suggested that the optimal therapeutic protocol was a repeated single infusion at the time of $\mathrm{B}$ cell recover $\mathrm{y}^{20)}$.
Our study showed the same excellent efficacy of RTX for SDNS as both maintenance and non-maintenance therapy as a recent randomized controlled trial ${ }^{11)}$. In the non-maintenance therapy, relapse rates were significantly decreased after a single dose of RTX. Patients and their parents did not want maintenance therapy because of anxiety about drug side effects. However, three patients in the non-maintenance group repeated two or more cycles because of frequent relapses. Most patients maintained remission for a significant period of time after recovery of the B cell count and two patients maintained long-term remission for 24 months. After non-maintenance therapy, some patients were likely to relapse less frequently than before RTX. RTX seems to reduce steroid dependency after treatment in some patients.

Thus, in non-maintenance therapy, when we decide to administer an additional cycle of RTX after relapse, we should carefully consider the frequency of relapse or steroid dependency. If relapse occurs infrequently, an additional cycle of RTX may not be needed.

Maintenance therapy in our study could maintain longterm remission without other immune-suppressants, especially corticosteroids. However, we cannot know for certain how long we should continue maintenance therapy. The practical regimen of RTX may require adjustment in an individual manner depending on the response and clinical condition in patients on non-maintenance or maintenance therapy. We suppose that maintenance therapy can be a good option to spare steroids for several months or years in patients with severe steroid toxicity.

The development of anti-RTX antibodies and human anti-chimeric antibodies (HACA) has been reported with RTX treatment in various disease ${ }^{6,721,22)}$; recently, the development of HACA in patients with NS who did not achieve $B$ cell depletion after repeated administration of RTX was reported by Ahn et al, a study in which two patients experienced a hypersensitivity reaction during a second infusion of RTX ${ }^{23)}$. Although drug side effects in our study did not increase as RTX cycles were repeated, we should carefully monitor drug side effects and B cell depletion, especially when cycles are repeated.

The most common side effect of RTX therapy was infusion-related hypersensitivity reaction such as fever and chills. However, the RTX infusion was completed in all 
patients by slowing down of the infusion rate and medications including antihistamines and corticosteroids. There have been very few reports about patients who developed severe side effects such as anaphylactic reactions, hypotension, arrhythmia, pulmonary fibrosis, and myocarditis ${ }^{15-}$ $17,24,25)$.

The limitations of this study include that it was retrospectively conducted at a single center, included a small sample size, and was not controlled.

In conclusion, RTX is an effective and safe option for maintaining remission in patients with SDNS without severe side effects as either maintenance or non-maintenance therapy. Larger samples in randomized clinical trials are needed to confirm the efficacy, safety, and standard regimen of RTX in patients with intractable NS.

\section{Conflicts of interest}

No potential conflict of interest relevant to this article was reported.

\section{Ethics statement}

The present study was reviewed and approved by the Institutional Review Board of Pusan National University Yangsan Hospital (IRB No. 05-2016-153).

\section{References}

1. Ehrich JHH, Geerlings C, Zivicnjak M, Franke D, Geerlings H, Gellermann J. Steroid-resistant idiopathic childhood nephrosis: overdiagnosed and undertreated. Nephrol Dial Transplant 2007; 22:2183-93.

2. Kyrieleis HAC, Löwik MM, Pronk I, Cruysberg HRM, Kremer JAM, Oyen WJG, et al. Long-term outcome of biopsy-proven, frequently relapsing minimal-change nephrotic syndrome in children. Clin J Am Soc Nephrol CJASN 2009;4:1593-600.

3. Guigonis V, Dallocchio A, Baudouin V, Dehennault M, Hachon-Le Camus C, Afanetti M, et al. RTX treatment for severe steroid- or cyclosporine-dependent nephrotic syndrome: a multicentric series of 22 cases. Pediatr Nephrol 2008;23:1269-79.

4. Bagga A, Sinha A, Moudgil A. RTX in patients with the steroidresistant nephrotic syndrome. N Engl J Med 2007;356:2751-2.
5. Gilbert RD, Hulse E, Rigden S. RTX therapy for steroid-dependent minimal change nephrotic syndrome. Pediatr Nephrol 2006;21: 1698-700.

6. Keystone E, Fleischmann R, Emery P, Furst DE, van Vollenhoven $R$, Bathon J, et al. Safety and efficacy of additional courses of RTX in patients with active rheumatoid arthritis: an open-label extension analysis. Arthritis Rheum 2007;56:3896-908.

7. Looney RJ, Anolik JH, Campbell D, Felgar RE, Young F, Arend LJ, et al. B cell depletion as a novel treatment for systemic lupus erythematosus: a phase I//I dose-escalation trial of RTX. Arthritis Rheum 2004;50:2580-9.

8. Benz K, Dötsch J, Rascher W, Stachel D. Change of the course of steroid-dependent nephrotic syndrome after RTX therapy. Pediatr Nephrol 2004;19:794-7.

9. Sinha A, Bhatia D, Gulati A, Rawat M, Dinda AK, Hari P, et al. Efficacy and safety of RTX in children with difficult-to-treat nephrotic syndrome. Nephrol Dial Transplant 2015;30:96-106.

10. Kemper MJ, Gellermann J, Habbig S, Krmar RT, Dittrich K, Jungraithmayr T, et al. Long-term follow-up after RTX for steroid-dependent idiopathic nephrotic syndrome. Nephrol Dial Transplant 2012;27:1910-5.

11. lijima K, Sako M, Nozu K, Mori R, Tuchida N, Kamei K, et al. RTX for childhood-onset, complicated, frequently relapsing nephrotic syndrome or steroid-dependent nephrotic syndrome: a multicentre, double-blind, randomised, placebo-controlled trial. Lancet 2014;384:1273-81.

12. Gulati A, Sinha A, Jordan SC, Hari P, Dinda AK, Sharma S, et al. Efficacy and safety of treatment with RTX for difficult steroid-resistant and -dependent nephrotic syndrome: multicentric report. Clin J Am Soc Nephrol 2010;5:2207-12.

13. Kaito H, Kamei K, Kikuchi E, Ogura M, Matsuoka K, Nagata M, et al. Successful treatment of collapsing focal segmental glomerulosclerosis with a combination of RTX, steroids and ciclosporin. Pediatr Nephrol 2010;25:957-9.

14. Magnasco A, Ravani P, Edefonti A, Murer L, Ghio L, Belingheri M, et al. RTX in children with resistant idiopathic nephrotic syndrome. J Am Soc Nephrol 2012;23:1117-24.

15. Cravedi P, Ruggenenti P, Sghirlanzoni MC, Remuzzi G. Titrating RTX to circulating B cells to optimize lymphocytolytic therapy in idiopathic membranous nephropathy. Clin J Am Soc Nephrol 2007;2:932-7.

16. Kamei K, Ito S, Nozu K, Fujinaga S, Nakayama M, Sako M, et al. Single dose of RTX for refractory steroid-dependent nephrotic syndrome in children. Pediatr Nephrol 2009;24:1321-8.

17. Fujinaga S, Hirano D, Nishizaki N, Kamei K, Ito S, Ohtomo Y, et al. Single infusion of RTX for persistent steroid-dependent minimalchange nephrotic syndrome after long-term cyclosporine. Pediatr Nephrol 2010;25:539-44.

18. Ito S, Kamei K, Ogura M, Udagawa T, Fujinaga S, Saito M, et al. Survey of RTX treatment for childhood-onset refractory nephrotic syndrome. Pediatr Nephrol 2013;28:257-64.

19. Niu X-L, Hao S, Wang P, Zhang W, Guo G-M, Wu Y, et al. Single 
dose of RTX in children with steroid-dependent minimal change nephrotic syndrome. Biomed Rep 2016:5:237-42.

20. Tellier S, Brochard K, Garnier A, Bandin F, Llanas B, Guigonis V, et al. Long-term outcome of children treated with rituximab for idiopathic nephrotic syndrome. Pediatr Nephrol 2013;28:911-8.

21. Cornec D, Tempescul A, Querellou S, Hutin P, Pers J-O, Jamin C, et al. Identification of patients with indolent B cell lymphoma sensitive to RTX monotherapy. Ann Hematol 2012;91:715-21.

22. Igarashi T, Kobayashi Y, Ogura M, Kinoshita T, Ohtsu T, Sasaki Y, et al. Factors affecting toxicity, response and progression-free survival in relapsed patients with indolent B-cell lymphoma and mantle cell lymphoma treated with RTX: a Japanese phase II study. Ann Oncol 2002;13:928-43.

23. Ahn YH, Kang HG, Lee JM, Choi HJ, Ha I-S, Cheong HI. Development of antiRTX antibodies in children with nephrotic syndrome. Pediatr Nephrol 2014;29:1461-4.

24. Chaumais M-C, Garnier A, Chalard F, Peuchmaur M, Dauger S, Jacqz-Agrain E, et al.Fatal pulmonary fibrosis after RTX administration. Pediatr Nephrol 2009;24:1753-5.

25. Sellier-Leclerc A-L, Belli E, Guérin V, Dorfmüller P, Deschênes $G$. Fulminant viral myocarditis after RTX therapy in pediatric nephrotic syndrome. Pediatr Nephrol 2013;28:1875-9. 